\title{
Studying the diffuse ultraviolet background radiation with tomography
}

\author{
R. C. Henry ${ }^{\star}$ \\ Center for Astrophysical Sciences, Henry A. Rowland Department of Physics and Astronomy, \\ The Johns Hopkins University, Baltimore, MD 21218, USA \\ Received 24 March 2003 / Accepted 12 August 2003

\begin{abstract}
Observation of faint diffuse ultraviolet background radiation is a delicate undertaking, given the brightness of the ultraviolet stars against which discrimination is essential. I demonstrate that the technique of tomography is unsuited to this particular task.
\end{abstract}

Key words. techniques: spectroscopic - instrumentation: spectrographs - ultraviolet: general

\section{Introduction}

Most observational astronomy focuses on either the observation of point sources, or on observation of small extended sources such as galaxies. Observation of true general diffuse ultraviolet background radiation requires instrumentation that is quite different from what is most effective for the observation of point sources.

Indeed, the whole field of diffuse ultraviolet background radiation study began with the use of inappropriate instrumentation, with unfortunate results (Lillie \& Witt 1976). Their use of instrumentation with slow optics (resulting in a very tiny field of view) meant that very small errors in dark current determination translated into very large errors in the deduced cosmic background radiation; and similarily, the presence of even a very faint star in the field of view, that is misinterpreted as diffuse background, results in a very large false background; and this false background of course would be expected to be patchy, and would be expected to increase toward lower galactic latitudes, where there are many more such faint stars.

The measurement of a diffuse background in the presence of stars is very difficult and has been marked by controversy (Bowyer 1991; Henry 1991). But only by fully understanding the defects of proposed methods, is progress possible.

\section{SPINR}

A recent attempt to measure the diffuse ultraviolet background in Scorpius is reported by Cook et al. (2003). Their "SPINR" rocket instrumentation uses fast optics, which is appropriate for the measurement of diffuse backgrounds, but SPINR has a severe problem, as we shall see, with discrimination of the

^ e-mail: henry@jhu.edu diffuse cosmic background against ambient starlight and geocoronal $\mathrm{L} \alpha$, which are admitted to their spectrographs throughout their observation.

The instrumentation of Cook et al. involves the use of a grating to image a $10^{\circ} \times 10^{\circ}$ region of the sky onto the detector throughout the observation. All the stars (as well as the background radiation and the geocoronal emission) within five degrees on each side of the entrance slit are imaged onto an image of the same slit, on the surface of the detector, and this occurs for every position along the slit. The instrument and detector provide spatial resolution of $5^{\prime}$ on the sky.

To allow an attempt at recovery of the spatial information in the second dimension, the slit (and, indeed, the whole instrument) is rotated on the sky, slowly, through $360^{\circ}$. The Cook et al. target in Scorpius (called by them "Scorpio") is mapped in Fig. 1. The result of their observation is their "sinogram," which I exhibit in Fig. 2. The techniques of tomography (Starck et al. 2002) are then used in the attempt to recover the original scene.

The degree of actual effectiveness of deconvolution may be judged pragmatically by examining the three different deconvolutions that are presented by Cook et al. (2003) using three different mathematical deconvolution methods. The result for the diffuse background (the scientific aim of the experiment) is very different in each of the three cases, and it is impossible to tell which of these, if any, is correct. This is due to the nature of tomography, which we now briefly investigate.

Once we have perused our primer on tomography, bringing out that technique's extreme vulnerability to noise, we will investigate the strong sources of noise that are actually present in the SPINR data, unfortunately rendering any meaningful observation of diffuse ultraviolet background radiation via that technique essentially impossible. 


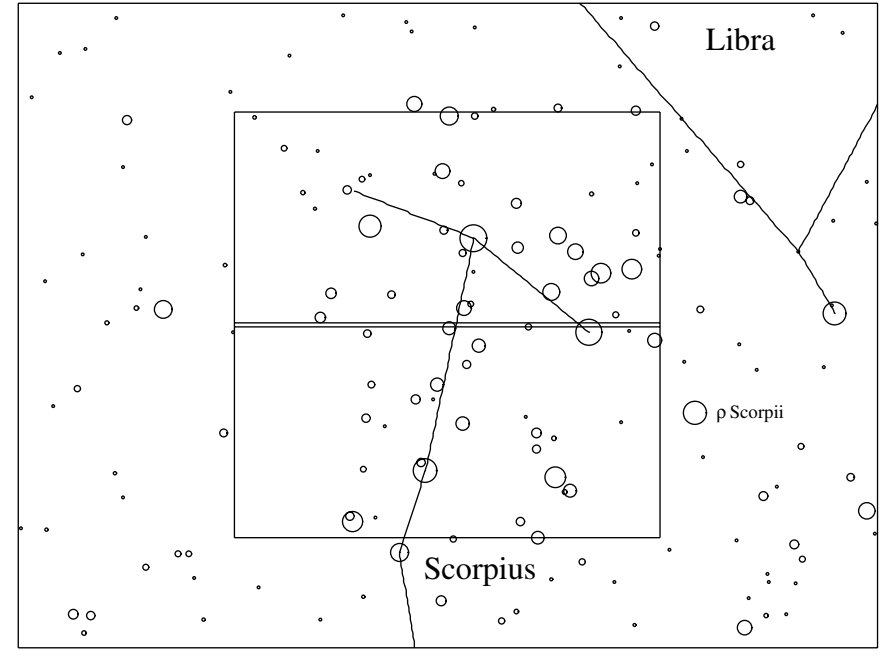

Fig. 1. The sky at $1565 \AA$ (from Henry et al. 1988), showing the $10^{\circ} \times 10^{\circ}$ field in Scorpius that was observed by Cook et al. (2003). All of the ultraviolet light in $5^{\prime}$ wide vertical stripes is imaged onto the narrow horizontal slit for spectroscopic dispersion. In the rocket's frame the sky slowly rotates around by $360^{\circ}$ as the rocket flight progresses. (The bright star $\rho$ Scorpii moves into, and out of, the field of view, as the rocket payload rotates.)

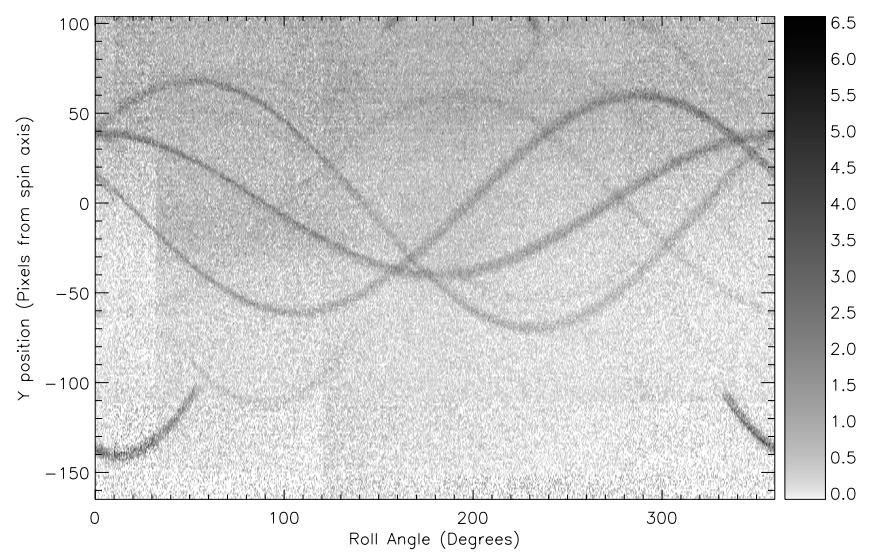

Fig. 2. This is the published SPINR rocket sinogram (Cook et al. 2003). The four dark vertical bands are identified (in the present paper) as being due to the star $\rho$ Scorpii as it moves on and off the grating.

\section{Basic tomography}

The literature on tomography is vast, and is complex. However, we can easily extract the essence of tomography by means of a very simple example, namely consideration of the tomographic measurement of a $2 \times 2$ - element region of the sky, the four patches having true brightnesses of 1000 counts, 100 counts, 10 counts, and 2 counts. We can imagine our target to be a star that is flanked by three faint patches of diffuse emission, measurement of the latter being our scientific goal. Perhaps the faintest patch is due to the cosmic web of baryons emitting $\mathrm{O}$ VI radiation.

In tomography, one does not measure our four patches individually. In Table 1, our four patches' brightnesses are designated $z_{i j}$ while the four observations by the tomographer are of the sums that are designated $t_{i j}$. The tomographer's task is to
Table 1. Absolute spectroscopy $\left(z_{i j}\right)$ vs. tomography $\left(t_{i j}\right)$.

\begin{tabular}{lll}
\cline { 1 - 2 }$z_{11}=1000$ & $z_{12}=100$ & $t_{11}=1100$ \\
$z_{21}=10$ & $z_{22}=2$ & $t_{12}=12$ \\
\cline { 1 - 2 }$t_{21}=1010$ & $t_{22}=102$ &
\end{tabular}

Table 2. Result of noise-free tomography.

\begin{tabular}{rrrr}
\hline \hline$z_{11}$ & $z_{12}$ & $z_{21}$ & $z_{22}$ \\
\hline 1010 & 90 & 0 & 12 \\
1009 & 91 & 1 & 11 \\
1008 & 92 & 2 & 10 \\
1007 & 93 & 3 & 9 \\
1006 & 94 & 4 & 8 \\
1005 & 95 & 5 & 7 \\
1004 & 96 & 6 & 6 \\
1003 & 97 & 7 & 5 \\
1002 & 98 & 8 & 4 \\
1001 & 99 & 9 & 3 \\
1000 & 100 & 10 & 2 \\
999 & 101 & 11 & 1 \\
998 & 102 & 12 & 0 \\
\hline
\end{tabular}

try to deduce the former from the latter. It is a matter of solving the following four equations, in four unknowns:

$$
\begin{aligned}
& t_{11}=z_{11}+z_{12} \\
& t_{12}=z_{21}+z_{22} \\
& t_{21}=z_{11}+z_{21} \\
& t_{22}=z_{12}+z_{22} .
\end{aligned}
$$

The solutions (plural) for the case of our simple example are:

$$
\begin{aligned}
& z_{11}=998+z_{22} \\
& z_{12}=102-z_{22} \\
& z_{21}=12-z_{22} \\
& z_{22} .
\end{aligned}
$$

The possible values of $z_{22}$ are, of course, 0 through 12 ; the 13 solutions are given in Table 2 . These 13 possible solutions do include the "true" solution, but there is no way to pick it out.

Tomographers must therefore rely on some additional information (which they call "priors") to select a solution. In this case, for example, our brightest patch might be due to a star that is of already known brightness (1000 counts), in which case the correct solution can be selected.

However, so far we have ignored statistical noise. The true values of $z_{i j}$ will of course be subject to statistical fluctuation, as will the tomographer's values of $t_{i j}$. Noise is said to be the bane of tomography. 
Table 3. Effect of Poisson noise on absolutely known values.

\begin{tabular}{rrrr}
\hline \hline$z_{11}$ & $z_{12}$ & $z_{21}$ & $z_{22}$ \\
\hline 1000 & 100 & 10 & 2 \\
973 & 80 & 10 & 1 \\
993 & 95 & 10 & 2 \\
1036 & 98 & 10 & 2 \\
987 & 98 & 12 & 5 \\
1020 & 103 & 16 & 3 \\
1029 & 107 & 9 & 2 \\
1003 & 90 & 10 & 0 \\
\hline
\end{tabular}

I generate poisson statistical noise (on any specific absolute number of counts) by using the poidev subroutine of Press et al. (1992), which, in turn, uses their excellent ranl to generate a uniform deviate. I used parameter idum $=-270$ in ranl. (My supplying idum means that anyone can duplicate my results in detail.)

Table 3 gives the result of seven attempts to measure $z_{i j}$ an absolute number of counts subject to statistical fluctuation, the first line being the true (absolute) result, which is supplied for comparison.

Now let us introduce similar poissonian noise into $t_{i j}$, our tomographic measurements. I ran 1000 cases, asking Mathematica to find the solutions. In 993 cases, Mathematica reported that the equations have no solution. In the remaining seven cases, there were multiple solutions. Forcing $z_{i j}=1000$ as our "prior" leads to the seven "solutions" that appear in Table 4.

The important thing, in considering the extraction of a "solution" from tomography, is not the cases that appear in Table 4, but the 993 cases for which there is no solution. (We will see, below, what the tomographer does in the face of this fundamental difficulty.) It is for that reason that I have not taken averages in Tables 3 and 4; the average means nothing in the latter case.

The seven cases for which a solution does exist, are produced purely by chance. One fundamental problem of tomography is that you are trying to get an answer to a question that you have not asked. The question you asked of nature, by making your tomographic measurements, was "what are the values of $t_{i j}$ ?" If, instead, you had made your measurements with an appropriate astronomical instrument, you would have obtained a (noisy) solution to the actual problem. But, then you would have been asking a different question of nature than the one that the tomographer actually asks.

It is instructive to repeat our tomographic exercise, but now taking $z_{11}=10000$ so as to represent permitting a brighter star to contribute to the tomographic measurements. Now "solutions" are rarer; in Table 5 are given the 10 non-null results from 3000 trials. The important (indeed, the vital) point to note is that this brighter star leaves a more severe trail of statistical noise, contaminating more severely the "measurements" of the diffuse background.
Table 4. Effect of Poisson noise on tomographic measurements.

\begin{tabular}{rrrrr}
\hline \hline$z_{11}$ & $z_{12}$ & $z_{21}$ & $z_{22}$ & Trial \\
\hline 1000 & 100 & 10 & 2 & \\
1000 & 111 & 30 & -21 & 206 \\
1000 & 58 & -34 & 48 & 330 \\
1000 & 117 & 6 & 4 & 464 \\
1000 & 87 & 17 & -1 & 478 \\
1000 & 100 & 28 & -10 & 494 \\
1000 & 103 & 6 & 3 & 752 \\
1000 & 91 & -1 & 11 & 846 \\
\hline
\end{tabular}

Table 5. The "Brighter Star" effect on tomographic measurements.

\begin{tabular}{rrrrr}
\hline \hline$z_{11}$ & $z_{12}$ & $z_{21}$ & $z_{22}$ & Trial \\
\hline 10000 & 100 & 10 & 2 & \\
10000 & 137 & 47 & -35 & 150 \\
10000 & 29 & -80 & 89 & 209 \\
10000 & 81 & 8 & 4 & 726 \\
10000 & 118 & 16 & -8 & 1030 \\
10000 & 113 & 29 & -18 & 1532 \\
10000 & 6 & -84 & 103 & 1681 \\
10000 & 17 & -82 & 94 & 1723 \\
10000 & -11 & -98 & 113 & 1806 \\
10000 & -79 & -175 & 183 & 2741 \\
10000 & 143 & 47 & -40 & 2947 \\
\hline
\end{tabular}

In the case of SPINR, even the brightest stars are trailed the full length of the slit, giving each star the opportunity to deposit statistical noise everywhere.

Note that there is nothing special about stars as sources of statistical noise. In particular, the cosmic web of baryons is, according to the modeling, very contrasty (hence, "web") and so would statistically self-contaminate via tomography. Thus, even if no noise were present, tomography could not provide scientifically valid measurements of the diffuse cosmic ultraviolet background radiation.

So, what does a tomographer actually $d o$, with a typical measurement? For a typical measurement, no solution exists. So, the tomographer seeks an approximate solution, using one (or more) of a host of techniques. For example, CLEAN, familiar to radio astronomers, uses the assumption that all of the signal is due to delta functions, to find an approximate solution. Diffuse emission (the object of our present interest) is assumed non-existent.

Of course my example, above, is only the simplest possible example of the principles of tomography, but it brings out clearly (and in a manner that I have not seen published elsewhere) precisely why tomography suffers from its fundamental difficulties. Of course in a realistic situation, there are many more elements on the sky, and the observations are made from many angles of rotation, and not just two. But that changes 


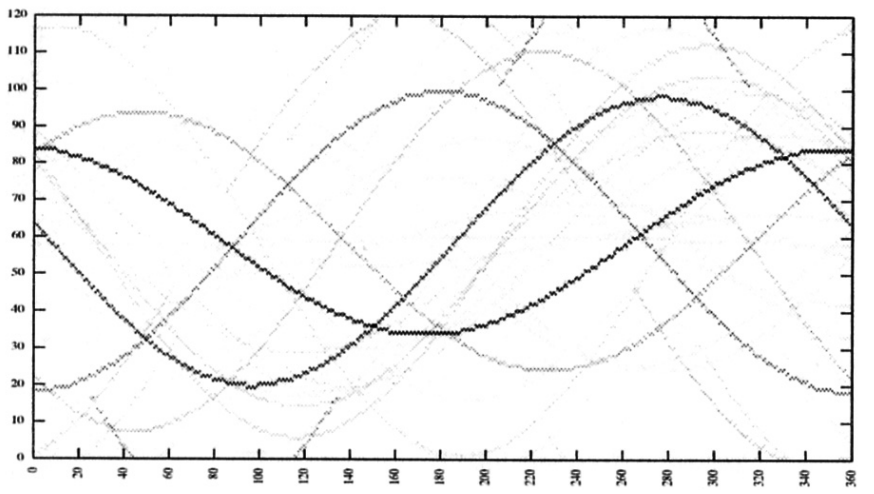

Fig. 3. This is my simulation of the SPINR Scorpius observation, on a linear intensity scale. There are 120 bins in the vertical direction, representing spatial resolution on the sky of 5 arcmin, and there are 360 slit positions on the sky, each rotated by one degree relative to its predecessor. Differences in brightnesses of individual stars between this simulation and the observation of Fig. 1 are undoubtedly due to the different wavelengths of the observation and of the simulation.

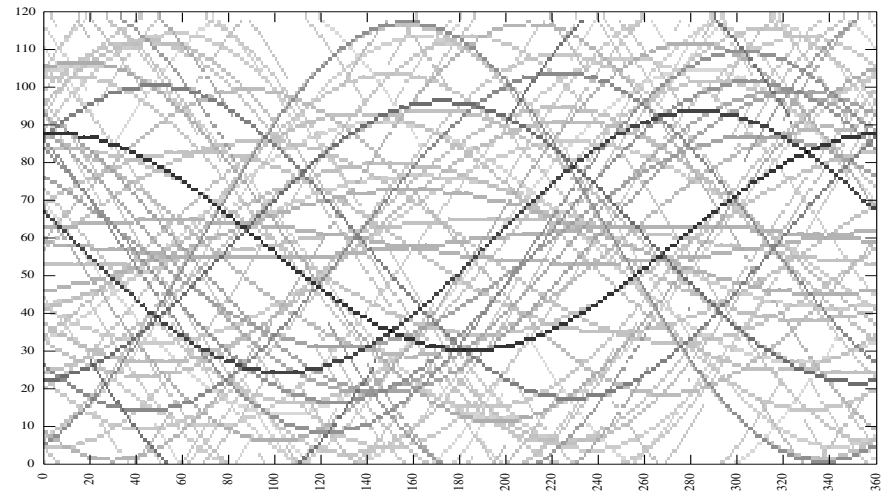

Fig. 4. This is the same simulation as in Fig. 3, but with a logarithmic intensity scale. This allows identification of patches that are uncontaminated with starlight, to the limit of the TD1 catalog. However, all such briefly-observed patches are subsequently contaminated through the tomographic data reduction process.

nothing in principle. In particular, if a bright star is present anywhere in the field of view, it will contaminate everywhere in a full rotation. The statistical noise cannot be removed, even in principle.

Tomographers are ingenious, and in the choice of approximation scheme, items such as insistence on positivity, for example, are often adopted. But that does not remove the errors, it simply makes them not obvious. Referring to the basic equation of tomography, Starck et al. (2002) say "Eq. (1) is usually in practice an ill-posed problem. This means that there is no unique and stable solution."

\section{Simulations}

I have carried out a simulation of the Scorpius observation of Cook et al. (2003) in order to assess the seriousness of stellar contamination. I used the TD1 Catalog (Henry et al. 1988 and references therein; this catalog may be obtained on $\mathrm{CD}$ from the National Space Science Data Center, at Goddard Space Flight Center) to simulate the stellar field. For each orientation of the slit on the sky, I simply determined which TD1 stars are within 5 degrees, for each $5^{\prime}$ segment of the slit, and summed their brightnesses at $1565 \AA$. There is no need to consider instrumental efficiencies; it is enough, for our present purposes, to simply determine how much light enters the experimental apparatus.

The resulting simulation is shown in Fig. 3, for the TD1 wavelength of $1565 \AA$. The result is clearly good general agreement. There is a very high background in Fig. 2 that the authors attribute to detector pressure problems. Differences in the brightnesses of individual stars are undoubtedly simply due to the difference in wavelength between the observation $(800$ to $1400 \AA$ ) and the simulation.

The linear plot of the simulation that is shown in Fig. 3 shows many more stars than are present in the observation, the fainter stars being undetectable in the latter case because of the noise background. In Fig. 4, I bring out more fully the number of TD1 stars that are contributing to the observation, by plotting my simulation result on a logarithmic intensity scale. This allows the reader to identify those regions (white patches) where no stellar contamination is present, at least down to the limit of the TD1 catalog.

By integrating the light of the stars in my simulation, I find that the total emission at $1565 \AA$ in the sinogram is 878000 photons $\mathrm{s}^{-1} \mathrm{~cm}^{-2}$. The amount of diffuse radiation that is present is unknown, but is much smaller than this direct starlight.

Of course the idea is that such stellar contamination does not matter, as the intention is to remove it by the deconvolution process. However, I have shown, above, that the stellar contamination can never be adequately removed, because of the severe effects of statistical noise.

Beyond the problem of photon noise, there are additional problems of instrumentally scattered starlight and geocoronal Lyman $\alpha$. The latter is ubiquitous, while the former is reduced for observations at high galactic latitudes. However, many people (for example, Bowyer 1991) feel that most of the diffuse ultraviolet background is due to starlight scattered from dust. If this is so, the ratio of diffuse radiation to starlight will be roughly the same everywhere on the sky, so avoidance of "bright" regions such as Scorpius and Orion has no effect on the present argument that the diffuse background cannot be successfully extracted (quite apart from the self-contamination problem mentioned above).

Before we turn to more quantitative assessment of the amount of noise that is present in the SPINR observation, we can crudely test for the actual presence of instrumental scattered light in that experiment (that is to say, in the data of Fig. 2) by means of my simulations: in my final simulation (Fig. 5) I demonstrate what character we would expect in the sinogram, as a result of any such instrumental scattered light. Simply in order to show the expected pattern, I allowed each star to contribute to the sinogram, not just at its appointed place along the slit, but by an additional and equal amount at $e v$ ery independent position along the slit. This of course represents vastly more noise than could be present due to scattered light - yet remarkably enough, dark bands appear in the simulation that are actually present in the data of Fig. 2. A little 
detective work (which consisted simply of one-at-a-time omission of the brightests stars from my simulation) showed that the star $\rho$ Scorpii (B2 IV-V, $V=3.88, B-V=-0.20$ ) is the star that, somehow, makes the bands as it repeatedly moves on and off the grating. It is clear that the SPINR data are massively contaminated with noise that is caused, one way or another, by the stars in the $10^{\circ} \times 10^{\circ}$ field of view of the instrument.

We are now well motivated to examine, more quantitatively, each of the sources of noise that might affect this (and other) SPINR attempts to measure the diffuse ultraviolet background radiation.

\section{Sources of noise affecting SPINR}

Starck et al. (2002) warn that "noise is the bane of the image analyst's life" and indicate that "unfortunately, however, deconvolution becomes a difficult problem due to the presence of noise in high-quality or deep imaging." Thus, noise is a concern that is of great seriousness to tomographers. In this section, we assess some of the sources of noise that are present in the SPINR observations.

Of course ultimately what one is interested in is signalto-noise, not noise itself. So let us start out by specifying the known (Bowyer 1991; Henry 1991; Schiminovich et al. 2001) brightness of the diffuse background radiation that is the object of scientific interest. At high galactic latitudes, the diffuse background longward of Lyman $\alpha$ is about 400 photon units, while the SPINR field of view is $10^{\circ} \times 10^{\circ}$ (or, $0.0305 \mathrm{sr}$ ), and its passband ( 900 to $1400 \AA$ ) is $500 \AA$. SPINR can thus expect 6100 photons $\mathrm{s}^{-1} \mathrm{~cm}^{-2}$ of true diffuse emission. We will, conservatively, use this number as a benchmark for comparison, although it is actually much too high, because the cosmic background drops sharply below Lyman $\alpha$ (Henry 1999). This will be our first entry, in our summary of the brightness of the ultraviolet sky as seen by SPINR (Table 6).

We have already considered one important source of noise, namely photon statistical fluctuations. In the case of radio astronomy (where typical $\lambda=21 \mathrm{~cm}$ ), in comparison with ultraviolet astronomy (for which typical $\lambda=1216 \times 10^{-8} \mathrm{~cm}$ ), there are 1727000 times as many photons per unit amount of energy, which is why photon noise is unimportant in radio tomography (and, why such photon noise is, as we have already seen, in contrast, so devastating to ultraviolet tomography).

The brightness of the ultraviolet sky was measured by Henry et al. (1980), and is identified by Henry (2002) as $1.252 \mathrm{ergs} \mathrm{s}^{-1} \mathrm{~cm}^{-2} \AA^{-1}$, corresponding to 120000 photons $\mathrm{s}^{-1} \mathrm{~cm}^{-2}$ over a $500 \AA$ passband and the SPINR field of view. This represents an average over the entire sky. I have determined, above, the brightness in the SPINR Scorpius target by integrating the brightnesses of all of the stars in Fig. 3, obtaining 878000 photons $\mathrm{s}^{-1} \mathrm{~cm}^{-2}$ entering the SPINR instrument. In Table 6, I have also entered the Orion region star brightnesses that are reported by Carruthers \& Opal (1977); the Orion region will be of interest to us below. Carruthers \& Opal also successfully measured the diffuse emission in Orion, and their number for that brightness is also given in Table 6. (Murthy et al. 2001 have reported Orion diffuse
Table 6. Brightness of the Ultraviolet Sky, as seen by SPINR.

\begin{tabular}{lllll}
\hline \hline Source & Sky mean & Scorpius & Orion & High lat \\
\hline Geo L $\alpha($ LEO) & 9700000 & 9700000 & 9700000 & 9700000 \\
Geo L $\alpha\left(5 R_{E}\right)$ & 970000 & 970000 & 970000 & 970000 \\
Starlight & 120000 & 878000 & 1540000 & 0 \\
Diffuse UV & 12000 & $(?)$ & 257000 & 6100 \\
O VI (galactic) & 79 & 150 & 150 & 150 \\
O VI (IGM) & $(?)$ & $(?)$ & $(?)$ & $(?)$ \\
\hline
\end{tabular}

radiation brightnesses that are similar to those of Carruthers \& Opal but at somewhat longer wavelengths.)

Henry (2002) showed that there is little diffuse galactic light in the ultraviolet, compared with direct starlight - his error bars suggest that $10 \%$ is the most that might be present. I have put that number in Table 6 as the "average" diffuse emission on the sky.

Finally, we must consider geocoronal emission at Lyman $\alpha$, which has intensity $4 \mathrm{kR}$ at rocket altitudes at night, declining to $0.4 \mathrm{kR}$ at 5 Earth radii (Rairden et al. 1986). Now, $4 \mathrm{kR}$ corresponds to 9700000 photons $\mathrm{s}^{-1} \mathrm{~cm}^{-2}$ entering an instrument with the SPINR field of view, and this number, and the corresponding number for observations made at 5 Earth radii, are also given in Table 6 .

\section{An additional problem: Grating scattering}

We have already demonstated the effects of statistical noise that arise because of the intrinsic nature of tomography. In addition, however, there are problems caused by light that is scattered at the grating. The SPINR detector subtends about $15^{\circ}$ at the grating, so moderate-angle scattered ultraviolet light will all end up on the detector, and will be re-distributed, of course entirely inappropriately, in the course of the tomographic analysis process.

Excellent gratings have an efficiency at the relevant wavelengths of about 0.5 (Osterman et al. 2000), which means that about half the photons do not go where one wishes them to go. Instead, they are sent to other orders; or they are absorbed; or they are scattered elsewhere - it is, of course, the last mentioned photons that are our present concern. Some fraction of these will scatter onto the detector at random positions. Subsequently, tomography will redistribute such scattered light in meaningless fashion, thereby contaminating all fainter emissions with noise.

Siegmund (2000) suggests that in the optical, $10 \%$ of all photons are scattered by contaminants at mirror surfaces at angles of about a degree (which in our present case would cause them to impact the SPINR detector). Cook et al. mention "losses due to contamination on the optical surfaces", but do not suggest where these lost photons end up. Consulting Table 6 , if even $1 \%$ of the Lyman $\alpha$ were scattered broadly, they would overwhelm the diffuse radiation at all galactic latitudes, and in addition, their statistical noise would add to the statistical uncertainty in the latter. 


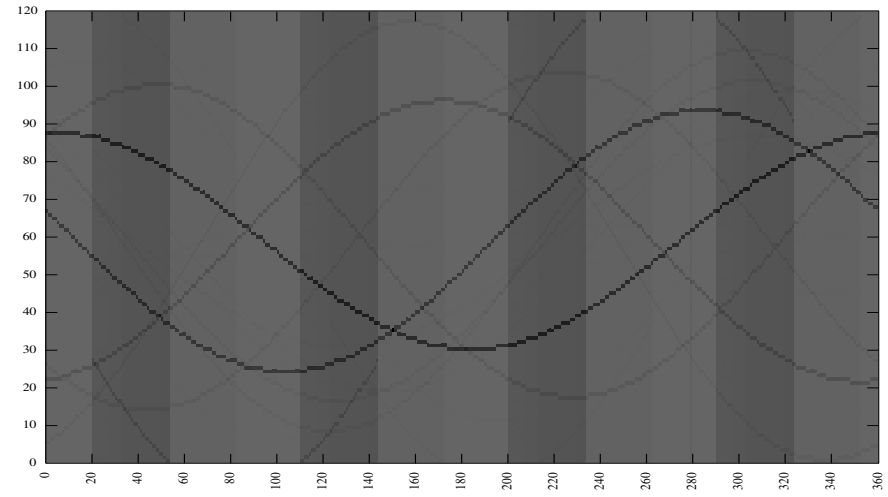

Fig. 5. In this final (linear) simulation, I have forced each star that is on the grating to scatter large amounts of light everywhere along the slit. The result is dark bands, where one particular star, $\rho$ Scorpii, moves on and off the grating repeatedly as the rocket rotates. Comparison with the actual sinogram (Fig. 2) proves that the rocket data are massively contaminated with instrumental noise. Instrumental scattered light is difficult to abate, since the essence of the technique requires the light of all the stars to strike a highly reflective grating that directly faces the detector.

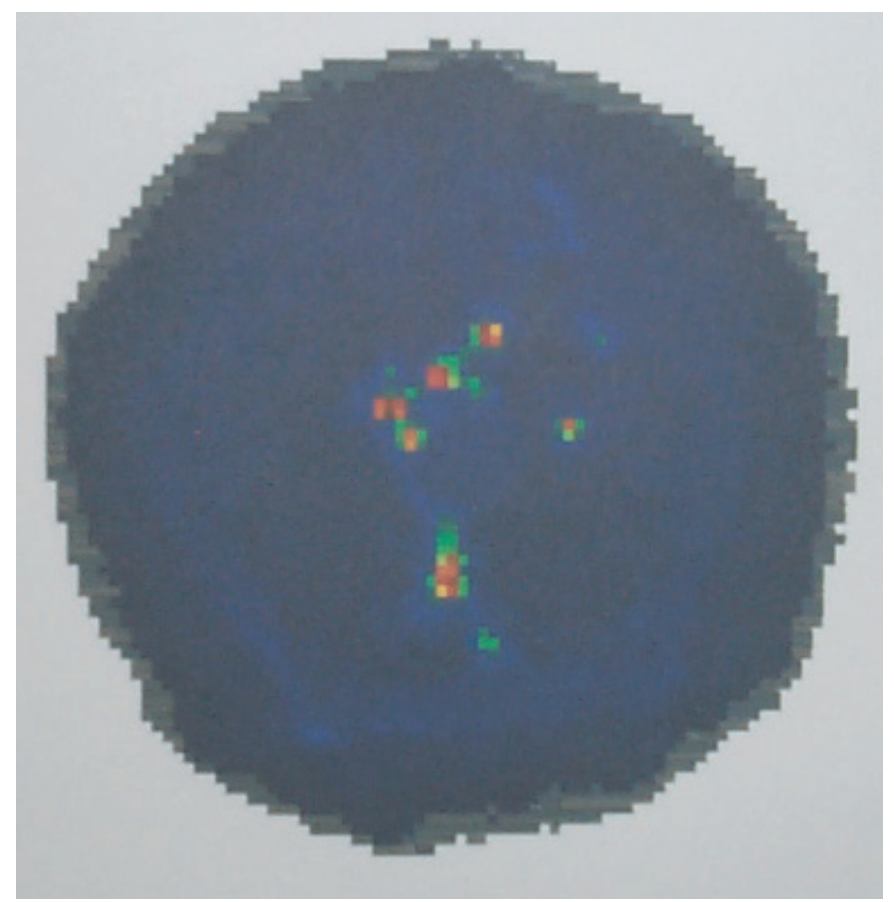

Fig. 6. De-convolved image of Orion at $1300 \AA$, from Cook et al. (2001).

Similarly, the scattering of starlight at the grating will make the effects of starlight substantially worse, even, than they would otherwise be, which (Table 6), is already bad.

In the light of our realization of the sensitivity of tomography to noise of any kind, examination of the data in Table 6, and in particular comparing the brightness of the signal that is sought, with the noise that is suggested by the other numbers in the table, indicates that tomography is not a technique that is appropriate to use in the measurement of diffuse ultraviolet background radiation brightnesses.

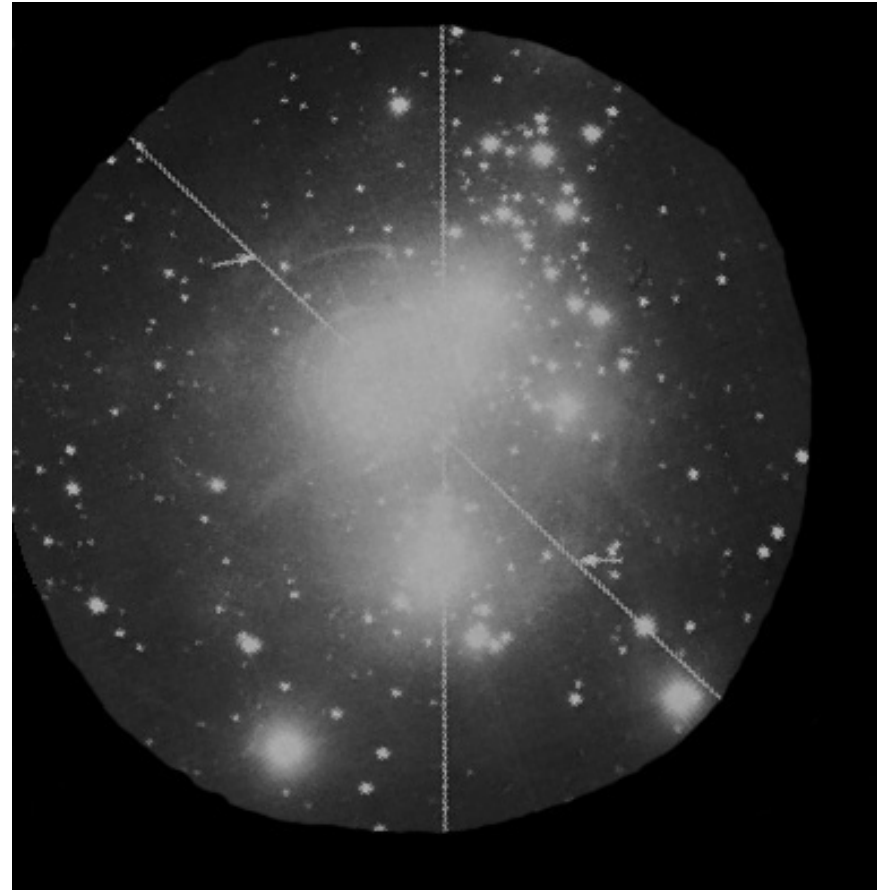

Fig. 7. Conventional mage of Orion (1230-2000 ̊̊), from Carruthers \& Opal (1977).

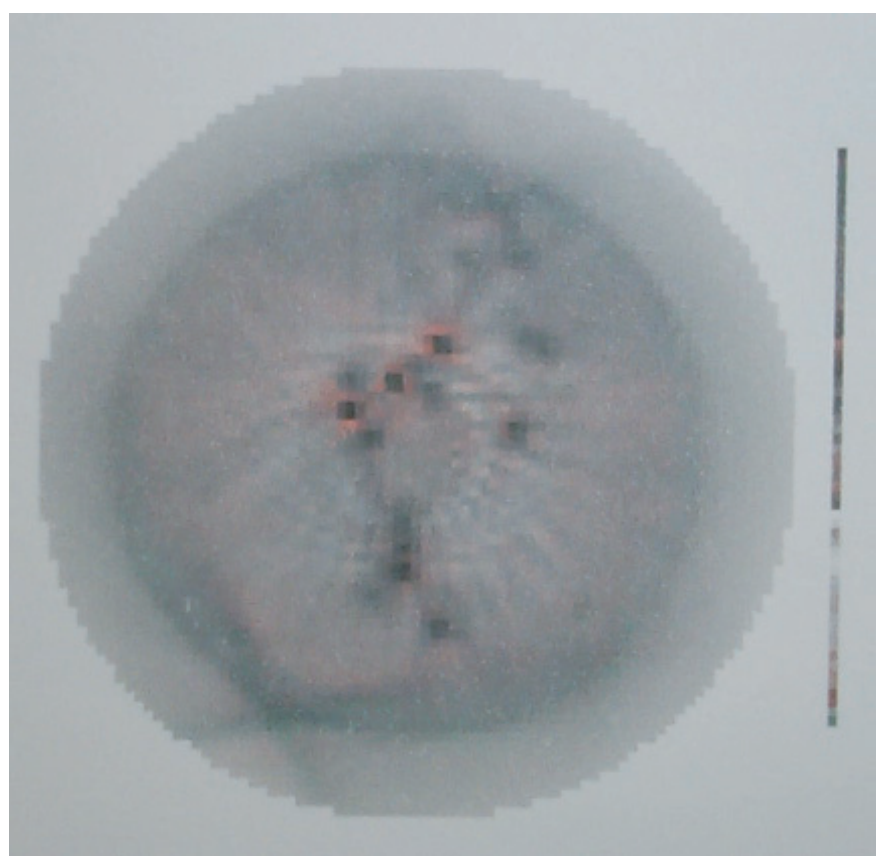

Fig. 8. Another de-convolved image of Orion, also from Cook et al. (2001). This is a three-color image (red $\equiv 1300-1400 \AA$, green $\equiv$ $1050-1150 \AA$, and blue $\equiv 900-1050 \AA$ ).

\section{Tomography of Orion}

The noise background that is reported by Cook et al. (2003) is attributed to pressure problems that would not be present normally. However, the same investigators also have successfully carried out a second rocket flight, in which no such problems occurred (Cook et al. 1999, 2000, 2001). In Fig. 6, I show their deconvolved image of Orion in the $1300 \AA$ band. It is 
instructive to compare this image with the conventional rocket ultraviolet image (Fig. 7) of Carruthers \& Opal (1977). There is no resemblance at all in the diffuse background: the Carruthers \& Opal image looks like any normal astronomical photograph, while the deconvolution exhibits a radial structure that indicates that it is dominated by artifacts.

Even more instructive (Fig. 8) is examination of the authors' combination of images, to form a single three color image. The authors indicate that "a large gamma correction is used to bring out the background features." Because such background features are precisely what we are most interested in, Fig. 8 is particularly important for developing understanding of what we can expect from tomography. We recall that "gamma correction" is a term originating in photography that, applied here to electronic images, indicates the deliberate and systematic distortion of intensities, with the constructive purpose of bringing out particularly important aspects of a given image. Russ (1995), in his Fig. 1.13, illustrate the precise meaning of "gamma:" "Gamma values greater than 1 expand the contrast range at the dark end of the range." The dark end of the range is, of course, in Fig. 8, the noise, plus the diffuse cosmic ultraviolet background radiation. Thus, by examining the image shown in Fig. 8, we can see the actual result of an attempt via tomography to study the diffuse ultraviolet background. But, what we see is a checkerboard pattern that strongly suggests a non-astrophysical origin for the bulk of the signal.

\section{Conclusion}

Spectroscopy of the diffuse ultraviolet background is important for many scientific reasons. One of the most important is the hope of direct detection of the baryonic intergalactic medium. One potentially important path to this is attempts to detect intergalactic $\mathrm{O}$ VI emission.

The final items in Table 6 are entries for the expected brightnesses of O VI emission from the galaxy, and from the intergalactic medium. There are no entries for the latter, because none appear to have been published. However, these brightnesses are surely substantially lower than the observed O VI emission from the interstellar medium. For the latter, the upper limit of Murthy et al. (2001) is entered in Table 6 as the "sky average," while the positive high-galactic-latitude detection by Shelton (2002), using FUSE, is given as what one might hope, perhaps, to see in Orion or Scorpio.
The O VI emission, from any source, is seen to be very faint. Thus, to successfully detect $\mathrm{O}$ VI emission (for example, in a search for emission from the intergalactic medium), one must use an approach that minimizes contamination by noise. In this paper, I have shown that tomography is not a viable approach to the achievement of this goal.

Acknowledgements. I thank the referee, Professor C. Stuart Bowyer, for his kind help in substantially improving the paper. This work was supported by Maryland Space Grant Consortium.

\section{References}

Bowyer, S. 1991, ARA\&A, 29, 59

Carruthers, G. R., \& Opal, C. B. 1977, ApJ, 212, L27

Cook, T. A., Taylor, V. J., \& Chakrabarti, S. 1999, Am. Astron. Soc. Meet. 195, \#88.09

Cook, T. A., Taylor, V. J., Golub, J., \& Chakrabarti, S. 2000, Am. Astron. Soc. Meet. 197, \#42.05

Cook, T. A., Golub, J., Taylor, V. J., \& Chakrabarti, S. 2001, Am. Astron. Soc. Meet. 199, \#59.02

Cook, T. A., Gsell, V. J., Golub, J., \& Chakrabarti, S. 2003, ApJ, 585, 1177

Henry, R. C. Anderson, R. C., \& Fastie, W. G. 1980, ApJ, 239, 859

Henry, R. C., Landsman, W. B., Murthy, J., et al. 1988, Atlas of the Ultraviolet Sky (Baltimore: The Johns Hopkins University Press)

Henry, R. C. 1991, ARA\&A, 29, 89

Henry, R. C. 1999, ApJ, 516, L49

Henry, R. C. 2002, ApJ, 570, 697

Lillie, C. F., \& Witt, A. N. 1976, ApJ, 208, 64

Murthy, J., Henry, R. C., Paxton, L., \& Price, S. D. 2001, Bull. Astr. Soc. India, 29, 563

Osterman, S., Wilkinson, E., Green, J. C., \& Redman, K. 2000, Proc. SPIE, 4013, 360

Press, W. H., Teukolsky, S. A., Vetterling, W. T., \& Flannery, B. P. 1992, Numerical Recipes in Fortran (Cambridge, New York: Cambridge University Press), 266

Rairden, R. L, Frank, L. A., \& Craven, J. D. 1986, JGR, 91, A12, 13613

Russ, J. C. 1995, The Image Processing Handbook, second ed. (Boca Raton: CRC Press)

Schiminovich, D., Friedman, P. G., Martin, C., \& Morrissey, P. F. 2001, ApJ, 563, L161

Shelton, R. 2002, ApJ, 569, 758

Siegmund, W. 2000, http://astro.Princeton.EDU/APO/ apo35-general/msg.78.html

Starck, J. L., Pantin, E., \& Murtagh, F. 2002, PASP, 114, 1051 